\title{
A NEW CHARACTERIZATION OF $B$-BOUNDED SEMIGROUPS WITH APPLICATION TO IMPLICIT EVOLUTION EQUATIONS
}

\author{
LUISA ARLOTTI
}

Received 22 September 2000

We consider the one-parameter family of linear operators that A. Belleni Morante recently introduced and called $B$-bounded semigroups. We first determine all the properties possessed by a couple $(A, B)$ of operators if they generate a $B$-bounded semigroup $(Y(t))_{t \geq 0}$. Then we determine the simplest further property of the couple $(A, B)$ which can assure the existence of a $C_{0}$-semigroup $(T(t))_{t \geq 0}$ such that for all $t \geq 0, f \in D(B)$ we can write $Y(t) f=T(t) B f$. Furthermore, we compare our result with the previous ones and finally we show how our method allows to improve the theory developed by Banasiak for solving implicit evolution equations.

\section{Introduction}

The study of the transport equation with multiplying boundary conditions recently prompted A. Belleni Morante to introduce a new class of evolution operators that he called $B$-bounded semigroups. The original definition of this class was given in [7] and generalized first by Belleni himself in [6], and then by Banasiak in [3]. Banasiak's definition reads as follows.

Definition 1.1. Let $X$ and $Z$ be Banach spaces and suppose that $A$ and $B$ are two linear operators such that

(i) $A: D(A) \rightarrow X, B: D(B) \rightarrow Z$, with $D(A), D(B) \subset X$,

(ii) $D(A) \subset D(B)$ and for some $\omega \in \mathbb{R}$ the resolvent set of $A$ satisfies $\rho(A) \supset$ ]$\omega, \infty[$.

A one-parameter family of linear operators $(Y(t))_{t \geq 0}$, which satisfies

(1) $Y(t): \Omega \rightarrow Z$, with $X \supset \Omega \supseteq D(B)$, and for any $t \geq 0$ and $f \in D(B)$

$$
\|Y(t) f\| \leq M \exp (\omega t)\|B f\|,
$$

(2) the function $t \rightarrow Y(t) f \in C([0, \infty[, Z)$ for any $f \in \Omega$,

Copyright $\odot 2000$ Hindawi Publishing Corporation Abstract and Applied Analysis 5:4 (2000) 227-244

2000 Mathematics Subject Classification: 47D06, 34G10

URL: http://aaa.hindawi.com/volume-5/S1085337500000336.html 
(3) for any $f \in \Omega_{0}=\{f \in D(A) ; A f \in \Omega\} \subset D(A) \subset D(B)$ and $t \geq 0$

$$
Y(t) f=B f+\int_{0}^{t} Y(s) A f d s
$$

is called a $B$-quasi bounded semigroup generated by $A$ and $B$.

Here and in what follows we denote by $\|\cdot\|$ the norm in the Banach space $Z$. In the case $B$ is bounded, $D(B)=X=Z, M=1, \omega=0$, one obtains the original definition, while the case $X=Z$ gives Belleni's generalization.

If the pair $(A, B)$ of operators generates a family satisfying conditions (1), (2), and (3) then we write $A \in B-G(M, \omega, X, Z)$ (or $A \in B-G(M, \omega, X)$, if $X=Z$ ).

Suppose that $A \in B-G(M, \omega, X)$.

It is immediate to see that, if $B \neq I, B \neq 0$ then the family $(Y(t))_{t \geq 0}$ is neither a $C_{0}$-semigroup nor an integrated semigroup $[1,9,10]$. In general, the family $(Y(t))_{t \geq 0}$ is not even a $C$-existence family [8]. A deep analysis of this topic, that is, on the link of $B$-bounded semigroups with $C$-existence families and $C$-regularized semigroups can be found in [5].

However, one can see that the notion of $B$-bounded semigroup is strictly related to the notion of empathy [4]. Furthermore, both the empathy theory and the $B$-bounded semigroup theory can be used for solving implicit evolution equations. In such a context the $B$-bounded semigroup method seems to generalize the method used by Showalter [11].

The above considerations show how important is to obtain a full characterization of generators of $B$-bounded semigroups. A first characterization, under the assumption that operators $A$ and $B$ satisfy all the conditions of Definition 1.1, has been obtained in [3]. For his study Banasiak introduced the extrapolation space $X_{B}$, which represents the completion of $X$ with respect to the (semi)norm $\|\cdot\|_{B}=\|B \cdot\|$. Later, without using the Banach space $X_{B}$, it has been proved in [2] that the Banasiak characterization theorem holds even if the assumption (ii) of Definition 1.1 is replaced by the weaker assumption:

(iii) there exists $\omega \in \mathbb{R}$ such that for each $\lambda>\omega$, the map

$$
(\lambda I-A): D_{B}(A) \longrightarrow D(B)
$$

where $D_{B}(A)=\{f \in D(A) \cap D(B): A f \in D(B)\}$, is bijective.

Recently, it has been shown that in many cases even this assumption is too restrictive and can be relaxed even further. The relaxation suggested in [5] is the following:

(iv) Denote by $A_{B}$ the part of $A$ in $D_{B}(A)$, that is, $A_{B}=\left.A\right|_{D_{B}}(A)$. We assume that the operator $A_{B}$ is closable in $X_{B}$, that is, we assume that if the sequence $\left(f_{n}\right)_{n \in \mathbb{N}}$ of elements of $D_{B}(A)$ is such that $B f_{n} \rightarrow 0$ and $B A f_{n} \rightarrow h$ in $Z$ as $n \rightarrow \infty$, then $h=0$. Denoting $\mathscr{T}=\bar{A}_{B}^{X_{B}}$, we assume further that there exist subspaces: $\mathscr{X}$ and $D$ satisfying $D(B) \subseteq \mathscr{X} \subseteq X_{B}$, and $D_{B}(A) \subseteq D \subseteq \mathscr{X} \cap D(\mathscr{T})$ such that $\left(\lambda I-\left.\mathscr{T}\right|_{D}\right): D \rightarrow \mathscr{X}$ is bijective for all $\lambda>\omega$.

In all the mentioned cases it has been proved that, if $A \in B-G(M, \omega, X, Z)$, then there exists a $C_{0}$-semigroup $(T(t))_{t \geq 0}$ in $Z_{B}=\overline{R(B)}$ such that $Y(t) f=T(t) B f$ for all $t \geq 0, f \in D(B)$. Such a semigroup is uniquely determined by the given pair $(A, B)$. 
In my opinion the achieved results are not completely satisfying yet. Indeed each paper contains assumptions on the pair $(A, B)$ which are weaker than those previously considered, but are sufficient to guarantee the existence in $Z_{B}$ of the above mentioned $C_{0}$-semigroup $(T(t))_{t \geq 0}$.

According to me the approach has to be completely reversed. In other words, first one has to determine all the properties possessed by a couple $(A, B)$ of operators if they generate a $B$-quasi bounded semigroup $(Y(t))_{t \geq 0}$. Then one has to determine which further assumption can assure that $Y(t) f=T(t) B f$ for all $t \geq 0, f \in D(B)$, where $(T(t))_{t \geq 0}$ is a $C_{0}$-semigroup in $Z_{B}$ which is uniquely determined by operators $A$ and $B$. It is the purpose of this paper to answer both questions.

In Section 2, we determine all the properties that two linear operators $A$ and $B$ possess if the following holds:

(a) assumption (i) of Definition 1.1 is satisfied;

(b) $A \in B-G(M, \omega, X, Z)$, that is, there exists a family $(Y(t))_{t \geq 0}$ such that conditions (1), (2), and (3) of Definition 1.1 hold;

(c) family $(Y(t))_{t \geq 0}$ satisfies the further condition

$$
Y(0) f=B f \quad \forall f \in D(B) .
$$

It is easy to see that assumption (c) is independent from assumptions (1), (2), and (3) of Definition 1.1, which only imply $Y(0) f=B f$ for all $f \in D_{B}(A)$. However, if we want to obtain the above-mentioned representation for the family $(Y(t))_{t \geq 0}$, then we have to suppose condition (c) be satisfied too.

In Section 3, we show that, if (a), (b), and (c) hold, then the simplest further condition that can assure the required representation of $(Y(t))_{t \geq 0}$ is the following:

(v) for all $f \in D(B), \lambda>\omega$ there exists a sequence $\left(f_{n}\right)_{n \in \mathbb{N}}$ of elements of $D_{B}(A)$ such that $B f_{n} \rightarrow g$ and $B A f_{n} \rightarrow h$ in $Z$ as $n \rightarrow \infty$ with $\lambda g-h=B f$.

In Section 4 we compare our result with the previous ones and show that condition (v) is weaker than (iv).

Finally, in Section 5 we show how our results allow to improve the theory developed in [4] for solving implicit evolution equations.

\section{Properties of a pair $(A, B)$ generating a $B$-bounded semigroup}

In this section, we want to characterize all the properties that two linear operators $A$ and $B$ possess if the above conditions (a), (b), and (c) are satisfied.

In order to achieve our aim, we consider the mapping $\mathscr{A}$ defined by putting, for all $g \in B\left(D_{B}(A)\right)$,

$$
\mathscr{A}(g)=\left\{h \in R(B): h=B A f \text { for } f \in D_{B}(A) \text { with } B f=g\right\} .
$$

Clearly the properties of mapping $\mathscr{A}$ and those of the pair $(A, B)$ are strictly linked with each other; indeed mapping $\mathscr{A}$ only depends on the given operators $A$ and $B$. Concerning this we can prove the following theorem. 
THEOREM 2.1. Let the operators $A$ and $B$ satisfy the conditions $(a),(b)$, and $(c)$. Then formula (2.1) defines a linear single-valued mapping $\mathscr{A}: B\left(D_{B}(A)\right) \rightarrow Z_{B}$, which is closable in $Z_{B}$. Its closure $\bar{A}$ has the following properties:

( $\alpha$ ) for all $\lambda>\omega$ one can define a linear bounded operator $R(\lambda): Z_{B} \rightarrow Z$ such that

$$
R(\lambda)(\lambda I-\bar{A}) g=g \quad \forall g \in D(\bar{A}),
$$

$(\beta)$ the mapping $(\lambda I-\bar{A}): D(\bar{A}) \rightarrow Z_{B}$ is injective for all $\lambda>\omega$,

$(\gamma) R(\lambda I-\bar{A})$ is a closed subset of $Z_{B}$ for all $\lambda>\omega$.

Remark 2.2. Observe that the statement $\mathscr{A}$ is a single-valued closable mapping in $Z_{B}$ is equivalent to the statement that the operator $A_{B}$ is closable in $X_{B}$. Indeed comparing (iv) with definition (2.1) one sees that both statements have the following meaning: if the sequence $\left(f_{n}\right)_{n \in \mathbb{N}}$ of elements of $D_{B}(A)$ is such that $g_{n}=B f_{n} \rightarrow 0$ and $\mathscr{A} g_{n}=$ $B A f_{n} \rightarrow h$ in $Z$ as $n \rightarrow \infty$, then $h=0$.

Proof of Theorem 2.1. It is known that the assumptions of Theorem 2.1 assure that formula (2.1) defines a linear single-valued mapping $\mathscr{A}: B\left(D_{B}(A)\right) \rightarrow Z_{B}$, which is closable in $Z_{B}$. Such a property has been proved in [1]. For the sake of completeness we report here the considerations contained in [1], but some of them in a slightly different form.

Consider $f \in D_{B}(A)$ such that $B f=0$. From properties of Definition 1.1 we obtain, for all $t \geq 0$,

$$
Y(t) f=0, \quad \int_{0}^{t} Y(s) A f d s=Y(t) f-B f=0 .
$$

But then $Y(t) A f=0$ for any $t \geq 0$. In particular, $B A f=Y(0) A f=0$. This shows that $A$ is a single valued mapping from $B\left(D_{B}(A)\right)$ to $Z_{B}$. The linearity is easily verified.

In order to show that $\mathscr{A}$ is closable in $Z_{B}$ observe that we can define a one-parameter family of linear operators $(T(t))_{t \geq 0}$ from $R(B)$ to $Z$ by putting, for $f \in D(B), t \geq 0$,

$$
T(t) B f=Y(t) f .
$$

Thanks to property (1) of Definition 1.1 we obtain for any $g=B f \in R(B), t \geq 0$

$$
\|T(t) g\| \leq M \exp (\omega t)\|g\| .
$$

Therefore, for each $t \geq 0$ operator $T(t)$ is bounded. By the extension principle, it can be uniquely extended to a bounded linear operator with domain $Z_{B}$. Denote by $T(t)$ the extension too. Then inequality (2.5) holds for all $t \geq 0, g \in Z_{B}$. Moreover, definition (2.4) and condition $Y(0)=B$ yield $T(0)=I$.

From property (2) of Definition 1.1 we deduce that, fixed $g \in R(B), t \rightarrow T(t) g$ is a continuous function of $t$ for $0 \leq t<\infty$. Inequality (2.5) allows to extend the property for any $g \in Z_{B}$. Thus for $g \in Z_{B}$ the function $t \rightarrow T(t) g \in C([0, \infty), Z)$.

Finally, as a consequence of property (3) of Definition 1.1 we have for all $g=B f \in$ $B\left(D_{B}(A)\right)$

$$
T(t) g=g+\int_{0}^{t} T(s) B A f d s,
$$


that is, thanks to $(2.1)$

$$
T(t) g=g+\int_{0}^{t} T(s) \mathscr{A} g d s .
$$

Now we are able to show that operator $\mathscr{A}$ is closable in $Z_{B}$. Indeed, consider a sequence $\left(g_{n}\right)_{n \in \mathbb{N}}$ of elements of $D(\mathscr{A})=B\left(D_{B}(A)\right)$ such that $g_{n} \rightarrow 0$ and $\mathscr{A} g_{n} \rightarrow h$ in $Z$ as $n \rightarrow \infty$. Clearly $h \in Z_{B}$ and $T(t) g_{n} \rightarrow 0, T(t) A_{A} g_{n} \rightarrow T(t) h$ for all $t \geq 0$ as $n \rightarrow \infty$. Now writing (2.7) for each $g_{n}$ and taking the limit as $n \rightarrow \infty$ we obtain for all $t \geq 0$,

$$
\int_{0}^{t} T(s) h d s=0 .
$$

Because this implies $T(t) h=0$ for all $t \geq 0$, and in particular $h=T(0) h=0$, operator $A$ is closable in $Z_{B}$.

Consider the closure $\bar{A}$ of $\mathscr{A}$. We remember that

$$
\begin{gathered}
D(\bar{A})=\left\{g \in Z_{B}: \exists\left(f_{n}\right)_{n \in \mathbb{N}} \text { in } D_{B}(A) \text { and } h \in Z_{B} \text { such that } B f_{n} \rightarrow g, B A f_{n} \rightarrow h\right\}, \\
\bar{A} g=\lim _{n \rightarrow \infty} B A f_{n} \quad \text { for } g=\lim _{n \rightarrow \infty} B f_{n} \in D(\bar{A}) .
\end{gathered}
$$

From this and formula (2.7) we immediately obtain for all $g \in D(\overline{\mathcal{A}}), t \geq 0$,

$$
T(t) g=g+\int_{0}^{t} T(s) \bar{A} g d s .
$$

Formula (2.11) allows to prove the stated properties.

$(\alpha)$ Put, for all $g \in Z_{B}, \lambda>\omega$,

$$
R(\lambda) g=\int_{0}^{+\infty} \exp (-\lambda t) T(t) g d t .
$$

Thanks to (2.5) we can state that for each $\lambda>\omega$ formula (2.12) defines a bounded linear operator from $Z_{B}$ to $Z$ with

$$
\|R(\lambda)\| \leq \frac{M}{\lambda-\omega} .
$$

From (2.11) and (2.12) we obtain, for all $\lambda>\omega, g \in D(\bar{A})$,

$$
\begin{aligned}
R(\lambda) \lambda g & =g+\lambda \int_{0}^{+\infty} \exp (-\lambda t) \int_{0}^{t} T(s) \bar{A} g d s d t \\
& =g+\int_{0}^{+\infty} \exp (-\lambda s) T(s) \bar{A} g d s=g+R(\lambda) \bar{A} g,
\end{aligned}
$$

that is $(2.2)$.

$(\beta)$ Suppose that $\lambda>\omega$ and $g \in D(\bar{A})$ such that $(\lambda I-\bar{A}) g=0$. From (2.2) and (2.12) we obtain $g=0$. This proves the assertion.

$(\gamma)$ Formulas (2.2) and (2.13) show that for any $\lambda>\omega$, the closed operator $(\lambda I-\bar{A})$ has bounded left inverse. Therefore its range is closed. This proves the statement. 
Remark 2.3. It is interesting to consider the case $X=Z$ and $B=I$.

Thanks to Theorem 2.1 in such a case we can state the following.

Suppose that $A: D(A) \rightarrow X$ is a linear operator in $X$. If there exists a one-parameter family of linear operators $(T(t))_{t \geq 0}$, which satisfies the following:

(1') $T(t): X \rightarrow X$, and for any $t \geq 0$ and $f \in X$,

$$
\|T(t) f\| \leq M \exp (\omega t)\|f\|,
$$

$\left(2^{\prime}\right)$ the function $t \rightarrow T(t) f \in C([0, \infty[, X)$ for any $f \in X$,

$\left(3^{\prime}\right)$ for any $f \in D(A)$ and $t \geq 0$

$$
T(t) f=f+\int_{0}^{t} T(s) A f d s,
$$

(4') $T(0)=I$,

then operator $A$ is closable. Its closure $\bar{A}$ has the following properties:

$\left(\alpha^{\prime}\right)$ for any $\lambda>\omega$, one can define a linear bounded operator $R(\lambda): X \rightarrow X$ such that

$$
R(\lambda)(\lambda I-\bar{A}) f=f \quad \text { for } f \in D(\bar{A}),
$$

$\left(\beta^{\prime}\right)$ the mapping $(\lambda I-\bar{A}): D(\bar{A}) \rightarrow X$ is injective for all $\lambda>\omega$,

$\left(\gamma^{\prime}\right) R(\lambda I-\bar{A})$ is a closed subset of $X$ for all $\lambda>\omega$.

\section{New characterization and generation theorems}

In Section 2, we have established which properties a pair $(A, B)$ of operators must possess if they generate a $B$-bounded semigroup, that is, if there exists a family $(Y(t))_{t \geq 0}$ which satisfies conditions (1), (2), and (3) of Definition 1.1. We have also shown that to each family $(Y(t))_{t \geq 0}$ satisfying conditions (1), (2), and (3) of Definition 1.1 we can associate a strongly continuous family of linear operators $(T(t))_{t \geq 0}$ from $Z_{B}$ to $Z$ so that the formula $T(t) B f=Y(t)$ for all $f \in D(B), t \geq 0$ holds. If furthermore $Y(0)=B$, then the family $(T(t))_{t \geq 0}$ is such that $T(0)=I$.

In this section, we first look for the simplest further property of the pair $(A, B)$ able to guarantee that the family $(T(t))_{t \geq 0}$ is a strongly continuous semigroup in $Z_{B}$. Considering (2.2) and (2.12), we immediately see that such a property can be obtained by requiring $\bar{A}$ to be the generator of a strongly continuous semigroup in $Z_{B}$ with the Hille constants $M$ and $\omega$, that is, $\bar{A} \in G\left(M, \omega, Z_{B}\right)$. Indeed, in such a case we have $T(t)=\exp (t \overline{\mathbb{A}})$. The study of the above-mentioned topic is carried out in Theorem 3.1.

Then we deduce a new generation theorem (Theorem 3.5) and a new characterization theorem (Theorem 3.6).

Now in order to prove Theorem 3.1 a preliminary consideration is necessary. Thanks to (2.9) and (2.10), condition (v) can be rewritten in the equivalent form:

for all $f \in D(B), \lambda>\omega$ there exists $g \in D(\bar{A})$ such that

$$
\lambda g-\bar{A} g=B f
$$


that is,

$$
R(\lambda I-\bar{A}) \supseteq R(B) \quad \forall \lambda>\omega .
$$

Considering this we can prove the following theorem.

Theorem 3.1. Suppose that all the assumptions of Theorem 2.1 are satisfied. Then $\bar{A} \in G\left(M, \omega, Z_{B}\right)$ if and only if the pair $(A, B)$ satisfies condition (v) too. In such a case we have for all $t \geq 0$,

$$
T(t)=\exp (t \bar{A})
$$

\section{Proof}

Necessity. Suppose that $\bar{A} \in G\left(M, \omega, Z_{B}\right)$. Then $R(\lambda I-\bar{A})=Z_{B}$ for all $\lambda>\omega$ and therefore $R(\lambda I-\bar{A}) \supseteq R(B)$ for all $\lambda>\omega$. But, as we know, such a property is equivalent to condition (v). Thus we can state that (v) is satisfied.

Sufficiency. Since $\bar{A}$ is a closed operator in $Z_{B}$ the Hille-Yosida theorem assures that $\bar{A} \in G\left(M, \omega, Z_{B}\right)$ if and only if the following conditions are satisfied:

(j) the resolvent set of $\bar{A}$ contains $] \omega,+\infty[$;

(jj) $\left\|(\lambda I-\bar{A})^{-n}\right\| \leq M /(\lambda-\omega)^{n}$ for all $\lambda>\omega$;

(jjj) $D(\bar{A})$ is dense in $Z_{B}$.

Now, we prove that all these conditions are satisfied whenever (v) holds.

(j) We know that assumption (v) is equivalent to condition (3.2). Since $R(\lambda I-\bar{A})$ is a closed subset of $Z_{B}$, we have

$$
R(\lambda I-\bar{A})=Z_{B} \quad \forall \lambda>\omega .
$$

This result, property $(\beta)$ of Theorem 2.1 and (2.2) show that for $\lambda>\omega$ the mapping

$$
(\lambda I-\bar{A}): D(\bar{A}) \longrightarrow Z_{B}
$$

is bijective with

$$
(\lambda I-\bar{A})^{-1}=R(\lambda)
$$

Now inequality (2.13) gives

$$
\left\|(\lambda I-\bar{A})^{-1}\right\| \leq \frac{M}{\lambda-\omega} .
$$

Therefore, $\rho(\overline{\mathscr{A}}) \supseteq] \omega,+\infty[$.

(jj) For any $\lambda>\omega, n \in \mathbb{N}$ and $g \in Z_{B}$ put

$$
R_{n}(\lambda) g=\frac{1}{(n-1) !} \int_{0}^{+\infty} \exp (-\lambda t) t^{n-1} T(t) g d t .
$$

The properties of the family $(T(t))_{t \geq 0}$ assure that for each $\lambda>\omega$ and $n \in \mathbb{N}$, formula (3.8) defines a bounded linear operator from $Z_{B}$ to $Z$ with

$$
\left\|R_{n}(\lambda)\right\| \leq \frac{M}{(\lambda-\omega)^{n}} .
$$


Therefore, we only have to prove that such an operator is given by

$$
R_{n}(\lambda)=(\lambda I-\bar{A})^{-n} .
$$

This can be shown by induction. Formula (3.8) holds for $n=1$ because (2.12) and (3.8) give

$$
R_{1}(\lambda)=R(\lambda)=(\lambda I-\bar{A})^{-1} .
$$

Suppose that formula (3.10) holds for $n=m$. Then we obtain from (2.11), for $\lambda>\omega$, $g \in D(\bar{A})$, that

$$
\begin{aligned}
R_{m+1}(\lambda) \lambda g= & \frac{1}{\lambda^{m}} g+\frac{\lambda}{m !} \int_{0}^{+\infty}\left(\int_{s}^{+\infty} t^{m} \exp (-\lambda t) d t\right) T(s) \bar{A} g d s \\
= & \frac{1}{\lambda^{m}} g+R_{m+1}(\lambda) \bar{A} g+\frac{1}{(m-1) !} \\
& \times \int_{0}^{+\infty}\left(\int_{s}^{+\infty} t^{m-1} \exp (-\lambda t) d t\right) T(s) \overline{\mathcal{A}} g d s .
\end{aligned}
$$

We easily deduce that

$$
R_{m+1}(\lambda) \lambda g=R_{m+1}(\lambda) \bar{A} g+R_{m}(\lambda) g
$$

that is,

$$
R_{m+1}(\lambda)(\lambda I-\bar{A}) g=R_{m}(\lambda) g=(\lambda I-\bar{A})^{-m} g .
$$

This shows that, if (3.10) holds for $n=m$, then it holds for $n=m+1$ too.

(jjj) In order to prove that $D(\bar{A})$ is dense in $Z_{B}$, it is sufficient to prove that

$$
\lim _{\lambda \rightarrow \infty} \lambda(\lambda I-\bar{A})^{-1} h=h \quad \forall h \in Z_{B}
$$

Note that

$$
\lambda(\lambda I-\bar{A})^{-1} h-h=\int_{0}^{\infty} \lambda \mathrm{e}^{-\lambda t}(T(t) h-h) d t .
$$

Because the function $t \rightarrow T(t) h$ is continuous at $t=0$, for any $\epsilon>0$ there exists $\delta>0$ such that for any $0 \leq t<\delta$,

$$
\|T(t) h-h\|<\epsilon .
$$

Therefore, for $\lambda>\omega_{1}=\max \{0, \omega\}$,

$$
\begin{aligned}
\left\|\lambda(\lambda I-\overline{\mathscr{A}})^{-1} h-h\right\| & \leq \int_{0}^{\infty} \lambda \mathrm{e}^{-\lambda t}\|T(t) h-h\| d t \\
& =\int_{0}^{\delta} \lambda \mathrm{e}^{-\lambda t}\|T(t) h-h\| d t+\int_{\delta}^{\infty} \lambda \mathrm{e}^{-\lambda t}\|T(t) h-h\| d t \\
& <\epsilon+2 M \int_{\delta}^{\infty} \lambda \mathrm{e}^{-\left(\lambda-\omega_{1}\right) t}\|h\| d t<2 \epsilon,
\end{aligned}
$$

for $\lambda$ sufficiently large. 
Thus we have shown that $\bar{A} \in G\left(M, \omega, Z_{B}\right)$.

Now, it is immediate to recognize that for any $t \geq 0$ operators $\exp (t \bar{A})$ and $T(t)$ coincide. Indeed, we know that for given $s \geq 0$ and $g \in D(\bar{A})$, we have $\exp (s \overline{\mathscr{A}}) g \in$ $D(\bar{A}) \subseteq Z_{B}$. Therefore, for each $t>0$ we can consider the function $s \rightarrow h(s), 0 \leq$ $s \leq t$ defined by

$$
h(s)=T(t-s) \exp (s \bar{A}) g .
$$

By virtue of formula (2.11) we have

$$
h^{\prime}(s)=-T(t-s) \overline{\mathscr{A}} \exp (s \overline{\mathscr{A}}) g+T(t-s) \overline{\mathscr{A}} \exp (s \overline{\mathscr{A}}) g=0 .
$$

This shows that $h(s)=h(0)$ for $0 \leq s \leq t$. In particular, we have $h(t)=h(0)$, that is,

$$
T(t) g=\exp (t \overline{\mathcal{A}}) g
$$

Because $t$ is an arbitrary positive number and $g$ is an arbitrary element of $D(\bar{A})$, which is dense in $Z_{B}$, formula (3.3) is proved for any $t \geq 0$.

Corollary 3.2. Suppose that all the assumptions of Theorem 3.1 are satisfied. Then for any $t \geq 0$ and $f \in D(B)$ we have $Y(t) f \in Z_{B}$.

Proof. If the assumptions of Theorem 3.1 hold, then for any $t \geq 0$ and $f \in D(B)$ we have

$$
Y(t) f=\exp (t \bar{A}) B f .
$$

But $\exp (t \bar{A})$ is a bounded linear operator in $Z_{B}$ for each $t \geq 0$. Therefore, $Y(t) f \in Z_{B}$ for any $t \geq 0$ and $f \in D(B)$.

Remark 3.3. Clearly Theorems 2.1 and 3.1 answer both the questions we asked in the introduction. In particular, Theorem 3.1 shows that the assumption (v) is the necessary and sufficient condition so that the representation (3.22) holds.

Now consider the following condition.

$\left(\mathrm{v}^{\prime}\right) B\left(D_{B}(A)\right)$ is dense in $Z_{B}$ and $R(\lambda I-\bar{A})=R(\mu I-\bar{A})$ for any $\lambda, \mu>\omega$. We can now prove the following proposition.

Proposition 3.4. If the assumptions of Theorem 2.1 are satisfied, then conditions (v) and $\left(v^{\prime}\right)$ are equivalent.

Proof. Suppose that the assumptions of Theorem 2.1 and condition (v) hold. Then also (3.4) holds and $R(\lambda I-\bar{A})=R(\mu I-\bar{A})$ for any $\lambda, \mu>\omega$. Moreover, thanks to point (jjj) of Theorem 3.1, we can state that $B\left(D_{B}(A)\right)$, which is dense in $D(\bar{A})$, is dense in $Z_{B}$ too. Thus we can conclude that condition $\left(\mathrm{v}^{\prime}\right)$ is satisfied.

Conversely, suppose that the assumptions of Theorem 2.1 and condition $\left(\mathrm{v}^{\prime}\right)$ hold. Then for given $\lambda, \mu>\omega$ and $f \in D(\bar{A})$ there exists $g \in D(\bar{A})$ such that

$$
(\lambda I-\bar{A}) g=(\mu I-\bar{A}) f
$$


and therefore,

$$
(\mu-\lambda) f=(\lambda I-\bar{A})(g-f) \in R(\lambda I-\bar{A}) .
$$

This shows that

$$
D(\bar{A}) \subseteq R(\lambda I-\bar{A})
$$

But then $R(\lambda I-\bar{A})$, which is a closed subset of $Z_{B}$, contains $\overline{D(\bar{A})}=\overline{B\left(D_{B}(A)\right)}=$ $Z_{B}$. This shows that condition (v) is satisfied.

Now we can state a new generation theorem in the following form.

Theorem 3.5. Let $X$ and $Z$ be Banach spaces and suppose that $A$ and $B$ are two linear operators such that

(i) $A: D(A) \rightarrow X, B: D(B) \rightarrow Z$, with $D(A), D(B) \subset X$.

The pair $(A, B)$ generates a $B$-quasi bounded semigroup $(Y(t))_{t \geq 0}$ with the property $Y(0) f=B f$ for all $f \in D(B)$, and satisfies either condition $(v)$ or condition $\left(v^{\prime}\right)$ if and only if (2.1) defines a single-valued mapping $\mathscr{A}: B\left(D_{B}(A)\right) \rightarrow Z_{B}$ which is closable in $Z_{B}$ and whose closure $\bar{A}$ generates a $C_{0}$-semigroup in $Z_{B}$. The $B$-quasi bounded semigroup $(Y(t))_{t \geq 0}$ is such that $Y(t) f=\exp (t \bar{A}) B f$ for any $t \geq 0$ and $f \in D(B)$.

Similarly we can state this new characterization theorem.

THEOREM 3.6. Let the operators $A$ and $B$ satisfy assumption $(i)$. Then the pair $(A, B)$ generates the $B$-quasi bounded semigroup $(Y(t))_{t \geq 0}$, with $Y(0)=B$, and satisfies either condition $(v)$ or condition $\left(v^{\prime}\right)$ if and only if $(2 . \overline{1})$ defines a single-valued mapping $\mathcal{A}: B\left(D_{B}(A)\right) \rightarrow Z_{B}$ which is closable in $Z_{B}$ and such that the following conditions hold:

(1) $B\left(D_{B}(A)\right)$ is dense in $Z_{B}$,

(2) there exist $M \geq 1$ and $\omega \in \mathbb{R}$ such that $\rho(\bar{A}) \supseteq] \omega,+\infty[$ and for any $f \in D(B)$, $\lambda>\omega$ and $n \in \mathbb{N}$ :

$$
\left\|(\lambda I-\bar{A})^{-n} B f\right\| \leq \frac{M}{(\lambda-\omega)^{n}}\|B f\| .
$$

Remark 3.7. Consider the case $X=Z$ and $B=I$.

By virtue of Theorem 3.1 we can state the following.

Suppose that there exists a one-parameter family of linear operators $(T(t))_{t \geq 0}$, which satisfies the conditions $\left(1^{\prime}\right),\left(2^{\prime}\right),\left(3^{\prime}\right)$, and $\left(4^{\prime}\right)$ of Remark 2.3. Then $\bar{A} \in G(M, \omega, X)$ if and only if either the condition

$$
R(\lambda I-\bar{A})=X \quad \forall \lambda>\omega
$$

or the condition

$$
\overline{D(A)}=X, \quad R(\lambda I-\bar{A})=R(\mu I-\bar{A}) \quad \text { for any } \lambda, \mu>\omega
$$

is satisfied. 


\section{Comparison with the previous results}

This section is devoted to compare our new characterization and generation theorems (Theorems 3.5 and 3.6) with Theorem 3.1 and Proposition 3.2 in [5].

To this aim we have to introduce the Banach space $X_{B}$ and to provide some results proved in [3].

Definition 4.1. We consider the set $\mathrm{X}$ of sequences $\left(f_{n}\right)_{n \in \mathbb{N}}$ such that $f_{n} \in D(B)$ for $n \in \mathbb{N}$ and $\left(B f_{n}\right)_{n \in \mathbb{N}}$ is a Cauchy sequence. We define $X_{B}$ to be the space of all classes of equivalence of sequences $\left(f_{n}\right)_{n \in \mathbb{N}} \in \mathrm{X}$ with respect to the following relation:

$$
\left(f_{n}\right)_{n \in \mathbb{N}} \equiv\left(g_{n}\right)_{n \in \mathbb{N}} \text { if and only if } \lim _{n \rightarrow \infty}\left\|B f_{n}-B g_{n}\right\|=0 .
$$

The space $X_{B}$ is a normed space with the norm

$$
\left\|\left[\left(f_{n}\right)_{n \in \mathbb{N}}\right]\right\|_{X_{B}}=\lim _{n \rightarrow \infty}\left\|B f_{n}\right\| .
$$

Definition 4.2. Denote by $\mathrm{P}$ the operator from $D(B)$ into $X_{B}$ defined by

$$
\mathrm{P} f=[(f, f, \ldots)] \text {. }
$$

Definition 4.3. Denote by $\mathscr{B}$ the linear operator from $X_{B}$ into $Z_{B}$ defined for $\Phi=$ $\left[\left(f_{n}\right)_{n \in \mathbb{N}}\right] \in X_{B}$ by

$$
\mathscr{B} \Phi=\lim _{n \rightarrow \infty} B f_{n} .
$$

It is known (see [3, Lemma 2.1 and Proposition 2.1]) that $\mathscr{B}$ is an isometric isomorphism of $X_{B}$ into $Z_{B}$.

We also recall the Banasiak characterization theorem proved in [5]. It states the following: suppose that the operators $A$ and $B$ satisfy assumption (i) of Definition 1.1 and assumption (iv). Then the pair $(A, B)$ generates a $B$-quasi bounded semigroup $(Y(t))_{t \geq 0}$, with $Y(0)=B$, if and only if the following conditions hold:

$\left(1^{\prime}\right) \mathscr{B}(D)$ is dense in $Z_{B}$,

$\left(2^{\prime}\right)$ there exist $M>0$ and $\omega \in \mathbb{R}$ such that for any $\eta \in \mathscr{L}, \lambda>\omega$, and $n \in \mathbb{N}$,

$$
\left\|\mathscr{B}\left(\lambda I-\left.\mathscr{T}\right|_{D}\right)^{-n} \eta\right\| \leq \frac{M}{(\lambda-\omega)^{n}}\|\mathscr{B} \eta\| .
$$

Finally, we recall that, according to Proposition 3.2 of [5], the family $(Y(t))_{t \geq 0}$ can be represented in the form

$$
Y(t)=\mathscr{B} \exp (t \mathscr{T}) \mathrm{P}
$$

if and only if the further following assumption is satisfied:

$\left(\mathrm{iv}^{\prime}\right)$ there exists some $\lambda>\omega$ such that the operator $\lambda I-\mathscr{T}$ is injective.

Now we can compare our new characterization theorem with the previous results. Such a comparison is carried out in the following propositions. 
Proposition 4.4. Condition (iv) implies condition (v).

Proof. Suppose that condition (iv) is satisfied. Consider $f \in D_{B}(A)$ such that $B f=0$ and take the sequence $\left(f_{n}\right)_{n \in \mathbb{N}}$ of elements of $D_{B}(A)$ such that $f_{n}=f$ for any $n$. Then $B f_{n}=B f=0$ and $B A f_{n}=B A f$ for any $n$. Since operator $A_{B}$ is closable in $X_{B}$, we have $B A f=0$. This shows that (2.1) defines a single-valued mapping from $B\left(D_{B}(A)\right)$ to $Z_{B}$. Moreover, considering Definition 4.3, we obtain

$$
\mathscr{A}=\mathscr{B} A_{B} \mathscr{B}^{-1} \text {. }
$$

The properties of the linear operator $\mathscr{T}_{B}$ assure that, if $A_{B}$ is closable in $X_{B}$, then $\mathscr{A}$ is closable in $Z_{B}$ with

$$
\begin{gathered}
D(\bar{A})=\mathscr{B}(D(\mathscr{T})), \\
\overline{\mathscr{A}}=\mathscr{B} \mathscr{T} \mathscr{B}^{-1},
\end{gathered}
$$

where, according to (iv), $\mathscr{T}=\bar{A}_{B}^{X_{B}}$.

We remember that

$$
\begin{aligned}
& D(\mathscr{T})=\{\Phi= {\left[\left(f_{n}\right)_{n \in \mathbb{N}}\right] \in X_{B}, \text { such that each } } \\
&\left.f_{n} \in D_{B}(A) \text { and }\left(B A f_{n}\right)_{n \in \mathbb{N}} \text { converges in } Z\right\}, \\
& \mathscr{T} \Phi=\left[\left(A f_{n}\right)_{n \in \mathbb{N}}\right] .
\end{aligned}
$$

Considering the properties of $\mathscr{B}$ and (4.10) we easily see that formulas (4.8) and (4.9) exactly give the operator defined by (2.10) and its domain (2.9), respectively. Now, assume that there exist subspaces $\mathscr{X}$ and $D$ satisfying $D(B) \subseteq \mathscr{X} \subseteq X_{B}$, and $D_{B}(A) \subseteq$ $D \subseteq \mathscr{X} \cap D(\mathscr{T})$ such that $\left(\lambda I-\left.\mathscr{T}\right|_{D}\right): D \rightarrow \mathscr{X}$ is bijective for all $\lambda>\omega$.

Then if we put

$$
\text { y }=\mathscr{B}(\mathscr{X}), \quad E=\mathscr{B}(D),
$$

we can state that there exist subspaces $\mathscr{Y}$ and $E$ satisfying $R(B) \subseteq \mathscr{Y} \subseteq Z_{B}$, and $D(\mathscr{A}) \subseteq E \subseteq \mathscr{Y} \cap D(\overline{\mathscr{A}})$ such that $\left(\lambda I-\left.\bar{A}\right|_{E}\right): E \rightarrow$ Oy is bijective for all $\lambda>\omega$. This implies that

$$
R(\lambda I-\bar{A}) \supseteq R\left(\lambda I-\left.\bar{A}\right|_{E}\right)=y \supseteq R(B),
$$

that is, condition $(\mathrm{v})$.

Proposition 4.5. Condition (1) of Theorem 3.6 and condition $\left(1^{\prime}\right)$ of Banasiak's theorem are equivalent.

Proof. Observe that $D(\mathscr{A})$ is dense in $D(\bar{A})$, with

$$
D(\mathscr{A})=B\left(D_{B}(A)\right) \subseteq \mathscr{B}(D) \subseteq D(\bar{A}) .
$$

Therefore

$$
\overline{B\left(D_{B}(A)\right)}=\overline{\mathscr{B}(D)}=\overline{D(\bar{A})} .
$$

This proves the statement. 
Proposition 4.6. Condition (2') of Banasiak's theorem implies Condition (2) of Theorem 3.6.

Proof. Using the operator $\mathscr{A}$, it is immediate to recognize that condition $\left(2^{\prime}\right)$ can be rewritten in the following equivalent form:

$\left(2^{\prime \prime}\right)$ there exist $M>0$ and $\omega \in \mathbb{R}$ such that for any $g \in \mathcal{Y}, \lambda>\omega$, and $n \in \mathbb{N}$,

$$
\left\|\left(\lambda I-\left.\bar{A}\right|_{E}\right)^{-n} g\right\| \leq \frac{M}{(\lambda-\omega)^{n}}\|g\| .
$$

But we know that $Y \supseteq R(B)$ and that $M$ cannot be less than 1 . Therefore, we deduce that if $\left(2^{\prime}\right)$ holds then condition (2) of Theorem 3.6 holds too.

Propositions 4.5 and 4.6 show that the assumptions made by Banasiak in [5] are stronger than ours.

Furthermore, suppose that the operators $A$ and $B$ satisfy the conditions (a), (b), and (c) of the introduction. Then property $(\beta)$ of Theorem 2.1 holds. By virtue of formula (4.9), we can rewrite that property in the equivalent form

$\left(\beta^{\prime}\right)$ the mapping $(\lambda I-\mathscr{T}): D(\mathscr{T}) \rightarrow X_{B}$ is injective for all $\lambda>\omega$.

This shows that condition (iv') is certainly satisfied if the pair $(A, B)$ of operators satisfies assumption (i) of Definition 1.1 and generates a $B$-quasi bounded semigroup $(Y(t))_{t \geq 0}$, with $Y(0)=B$. But then we can state that the assumptions of Theorem 3.1 in [5] imply $\left(\mathrm{iv}^{\prime}\right)$ and therefore they are sufficient to guarantee the representation (4.6). Such a property holds however we choose the set $D$. Finally, using (4.9) and Definition 4.2, we can easily see that the two representations (3.22) and (4.6) coincide. On the other hand, if the assumptions of our characterization theorem allow to write the family $(Y(t))_{t \geq 0}$ in the form (3.22), the stronger assumptions of the Banasiak characterization theorem must give the same representation, that is, (4.6), whatever the set $D$ is.

\section{Application to implicit evolution equations}

Recently Banasiak, [4], proved that the $B$-bounded semigroups theory can be applied for solving implicit evolution equations. Here we want to show how our technique can simplify such an application.

Indeed consider the Cauchy problem for the implicit evolution equation

$$
\frac{d}{d t}(K u)=L u, \quad \lim _{t \rightarrow 0^{+}}(K u)(t)=u_{0},
$$

where $K$ and $L$ are linear operators, $K: D(K) \rightarrow X$ and $L: D(L) \rightarrow X$, with $D(K)$, $D(L) \subseteq Z$. Here $X$ is a linear space and $Z$ is a Banach space.

Assume that the operator $K$ is one-to-one. Define $B=K^{-1}$ and to such an operator associate the Banach space $Z_{B}=\overline{R(B)}=\overline{D(K)}$. To $B$ associate also the Banach space $X_{B}$ and the operators $\mathrm{P}$ and $\mathscr{B}$ given by Definitions $4.1,4.2$, and 4.3 , respectively. If operators $K$ and $L$ are closable in $X_{B}$, then, according to [4], we introduce the following definition. 
Definition 5.1. A $Z$-valued function $t \rightarrow u(t)$ is called an $X_{B}$-solution to problem (5.1) if it is a classical solution of the problem

$$
\frac{d}{d t} \bar{K}^{X_{B}} u=\bar{L}^{X_{B}} u, \quad \lim _{t \rightarrow 0^{+}}\left(\bar{K}^{X_{B}} u\right)(t)=\mathrm{P} u_{0},
$$

that is, $t \rightarrow \bar{K}^{X_{B}} u(t)$ is continuously differentiable in $X_{B}$, the differential equation holds for all $t>0$ in $X_{B}$, and the initial condition holds as a limit in the topology of $X_{B}$.

Sufficient conditions for the existence of an $X_{B}$-solution to problem (5.1) are obtained in the following theorem.

Theorem 5.2. Assume that $K$ and $L$ are linear operators, $K: D(K) \rightarrow X$ and $L$ : $D(L) \rightarrow X$, where $D(K), D(L) \subseteq Z, X$ is a linear space and $Z$ a Banach space.

Assume that the operator $K$ is one-to-one. Define the operator $\mathscr{A}=K^{-1} L$ with the domain $D(\mathscr{A})=\{g \in D(K) \cap D(L): L g \in R(K)\}$. If $\mathscr{A}$ is closable in $Z_{B}$ and if $\bar{A} \in G\left(M, \omega, Z_{B}\right)$, then for any $u_{0} \in R(K)$ such that $K^{-1} u_{0} \in D(\mathscr{A})$, the function $t \rightarrow \exp (t \bar{A}) K^{-1} u_{0}$ is an $X_{B}$-solution to problem (5.1).

In order to prove such a theorem some preliminary considerations are necessary. First of all we can remark the following.

Remark 5.3. Given a sequence $\left(f_{n}\right)_{n \in \mathbb{N}}$ of elements of $D(B)=R(K)$, we can state that $\left(\mathrm{P} f_{n}\right)_{n \in \mathbb{N}}$ converges to $\Phi$ in $X_{B}$ as $n \rightarrow \infty$ if and only if $\left(K^{-1} f_{n}\right)_{n \in \mathbb{N}}$ converges to $\mathscr{B} \Phi$ in $Z_{B}$ as $n \rightarrow \infty$.

Now we have to show that the following propositions hold.

Proposition 5.4. If the operator $K$ is one-to-one, then it is closable in $X_{B}$, with

$$
D\left(\bar{K}^{X_{B}}\right)=Z_{B}, \quad \bar{K}^{X_{B}}=\mathscr{P}^{-1} .
$$

Proof. Take $g \in Z_{B}$. Because $\overline{D(K)}=Z_{B}$ there exists a sequence $\left(g_{n}\right)_{n \in \mathbb{N}}$ of elements of $D(K)$ such that $g_{n} \rightarrow g$ in $Z_{B}$ as $n \rightarrow \infty$.

Put $f_{n}=K g_{n}$ and $\Phi=\mathscr{B}^{-1} g$. Then $K^{-1} f_{n} \rightarrow \mathscr{B} \Phi$ in $Z_{B}$ as $n \rightarrow \infty$, and therefore, according to Remark 5.3, $\mathrm{P} K g_{n}=\mathrm{P} f_{n} \rightarrow \Phi=\mathscr{B}^{-1} g$ in $X_{B}$ as $n \rightarrow \infty$.

This proves the statement, since $g \in D\left(\bar{K}^{X_{B}}\right)$ and $\bar{K}^{X_{B}} g=\mathscr{B}^{-1} g$.

Proposition 5.5. Suppose that the operator $K$ is one-to-one and that the operator $A$ is closable in $Z_{B}$. Then $\left.L\right|_{D(\mathscr{A})}$ is closable in $X_{B}$, with

$$
D\left(\bar{L}^{X_{B}}\right)=D(\overline{\mathscr{A}}), \quad \bar{L}^{X_{B}}=\mathscr{B}^{-1} \overline{\mathscr{A}}
$$

Proof. Consider a sequence $\left(f_{n}\right)_{n \in \mathbb{N}}$ of elements of $D(\mathscr{A})$ such that $f_{n} \rightarrow 0$ in $Z_{B}$ and $\mathrm{P} L f_{n} \rightarrow \Phi$ in $X_{B}$ as $n \rightarrow \infty$. Then, according to Remark 5.3, $K^{-1} L f_{n} \rightarrow \mathscr{B} \Phi$ in $Z_{B}$ 
as $n \rightarrow \infty$. Because $\mathscr{A}=K^{-1} L$ is supposed to be closable in $Z_{B}$, we have $\mathscr{B} \Phi=0$ and therefore $\Phi=0$. This shows that $\left.L\right|_{D(\mathscr{A})}$ is closable in $X_{B}$. Now

$$
\begin{aligned}
D\left(\bar{L}^{X_{B}}\right) & =\left\{g \in Z_{B}: \exists\left(g_{n}\right)_{n \in \mathbb{N}} \text { in } D(\mathscr{A}) \text { and } \Phi \in X_{B}: g_{n} \rightarrow g, \mathrm{P} L g_{n} \rightarrow \Phi\right\} \\
& =\left\{g \in Z_{B}: \exists\left(g_{n}\right)_{n \in \mathbb{N}} \text { in } D(\mathscr{A}) \text { and } \Phi \in X_{B}: g_{n} \rightarrow g, K^{-1} L g_{n} \rightarrow \mathscr{B} \Phi\right\} \\
& =D(\bar{A}), \\
\bar{L}^{X_{B}} g & =\Phi=\mathscr{\mathscr { B }}^{-1} \overline{\mathscr{A}} g .
\end{aligned}
$$

Now we are able to prove Theorem 5.2.

Proof of Theorem 5.2. If the assumptions of Theorem 5.2 are satisfied then Propositions 5.4 and 5.5 hold. Suppose that $u_{0} \in R(K)$ such that $K^{-1} u_{0} \in D(\mathscr{A})$ and consider the function $t \rightarrow u(t)=\exp (t \overline{\mathscr{A}}) K^{-1} u_{0} \in D(\bar{A})$. Then the function $t \rightarrow \bar{K}^{X_{B}} u(t)=$ $\mathscr{B}^{-1} u(t)$ is continuously differentiable in $X_{B}$, with

$$
\frac{d}{d t} \bar{K}^{X_{B}} u(t)=\mathscr{B}^{-1} \overline{\mathscr{A}} \exp (t \bar{A}) K^{-1} u_{0}=\mathscr{B}^{-1} \overline{\mathscr{A}} u(t)
$$

By virtue of Proposition 5.5, we obtain for all $t>0$,

$$
\frac{d}{d t} \bar{K}^{X_{B}} u(t)=\bar{L}^{X_{B}} u(t)
$$

Moreover, taking into account definitions (4.7) and (4.8), we have

$$
\lim _{t \rightarrow 0^{+}}\left(\bar{K}^{X_{B}} u\right)(t)=\mathscr{B}^{-1} K^{-1} u_{0}=\mathrm{P} u_{0} .
$$

Thus the assertion is completely proved.

Remark 5.6. Suppose that the assumptions of Theorem 5.2 are satisfied and consider the family $(Y(t))_{t \geq 0}$ defined by putting for $f \in R(K), t \geq 0$

$$
Y(t) f=\exp (t \bar{A}) K^{-1} f .
$$

Such a family has the properties (1) and (2) of Definition 1.1. Moreover, for all $t>0$ and $f \in K(D(\mathscr{A}))$, we have

$$
\begin{aligned}
\frac{d}{d t} Y(t) f & =\exp (t \overline{\mathscr{A}}) \overline{\mathscr{A}} K^{-1} f=\exp (t \overline{\mathscr{A}}) \mathscr{A} K^{-1} f \\
& =\exp (t \overline{\mathscr{A}}) K^{-1} L K^{-1} f=Y(t) A f
\end{aligned}
$$

if we define $A=L K^{-1}$ with the natural domain

$$
D(A)=\left\{f \in R(K): K^{-1} f \in D(L)\right\}=K(D(K) \cap D(L)) .
$$


Clearly the above relation immediately gives, for all $t>0, f \in K(D(\mathscr{A}))$,

$$
Y(t) f=K^{-1} f+\int_{0}^{t} Y(s) A f d s,
$$

that is,

$$
Y(t) f=B f+\int_{0}^{t} Y(s) A f d s .
$$

Observing that

$$
\begin{aligned}
K(D(\mathscr{A})) & =\left\{f \in K(D(K) \cap D(L)): L K^{-1} f \in R(K)\right\} \\
& =\{f \in D(A): A f \in D(B)\}=D_{B}(A),
\end{aligned}
$$

we see that also property (3) of Definition 1.1 is satisfied with $A=L K^{-1}$. Therefore, the family $(Y(t))_{t \geq 0}$ is a $B$-quasi bounded semigroup generated by the pair $(A, B)$. Moreover, the relation between such a pair and the operator $\mathscr{A}$ is the same as in Section 4.

Remark 5.7. It is interesting to compare our Theorem 5.2 with Theorem 3.3 in [4]. Using the notation introduced in Section 4 and taking into account the characterization theorem proved in [5], we can write the content of Theorem 3.3 in [4] in the following way. Define operator $\mathscr{A}$ as in our Theorem 5.2. If $\mathscr{A}$ is closable in $Z_{B}$ and if there exists a subspace $E: D(\mathscr{A}) \subseteq E \subseteq D(\bar{A})$ such that $\left.\bar{A}\right|_{E}$ generates a $C_{0}$-semigroup $T(t)$ in $Z_{B}$, then for any $f \in D(A)$ the function $t \rightarrow T(t) K^{-1} f$ is an $X_{B}$-solution to problem (5.1). But, according to the considerations developed in Section 4 , if $\left.\bar{A}\right|_{E} \in G\left(M, \omega, Z_{B}\right)$, then $\bar{A}$ is a generator and $T(t)=\exp (t \bar{A})$. Thus the statement of our Theorem 5.2 and that of Banasiak's theorem (Theorem 3.3) coincide.

\section{References}

[1] W. Arendt, Vector-valued Laplace transforms and Cauchy problems, Israel J. Math. 59 (1987), no. 3, 327-352. MR 89a:47064. Zbl 637.44001.

[2] L. Arlotti, On B-bounded semigroups as a generalization of $C_{0}$-semigroups, $Z$. Anal. Anwendungen 19 (2000), no. 1, 23-34. CMP 1748056.

[3] J. Banasiak, Generation results for B-bounded semigroups, Ann. Mat. Pura Appl. (4) 175 (1998), 307-326.

[4] _ B-bounded semigroups and implicit evolution equations, Abstr. Appl. Anal. 5 (2000), no. 1, 13-32. CMP 1821727.

[5] J. Banasiak and V. Singh, B-bounded semigroups and $C$-evolution families, to appear.

[6] A. Belleni-Morante, On Some Properties of B-Quasi Bounded Semigroups and Applications, Dipartimento di Ingegneria Civile, Univerisitá di Firenze, quaderno sezione modelli matematici no. 1/1996.

[7] _ B-bounded semigroups and applications, Ann. Mat. Pura Appl. (4) 170 (1996), 359-376. MR 98c:47049. Zbl 882.47012.

[8] R. deLaubenfels, Existence Families, Functional Calculi and Evolution Equations, Lecture Notes in Mathematics, vol. 1570, Springer-Verlag, Berlin, 1994. MR 96b:47047. Zbl 811.47034. 
[9] H. Kellerman and M. Hieber, Integrated semigroups, J. Funct. Anal. 84 (1989), no. 1, 160180. MR 90h:47072. Zbl 689.47014.

[10] F. Neubrander, Integrated semigroups and their applications to the abstract Cauchy problem, Pacific J. Math. 135 (1988), no. 1, 111-155. MR 90b:47073. Zbl 675.47030.

[11] R. E. Showalter, Monotone Operators in Banach Space and Nonlinear Partial Differential Equations, Mathematical Surveys and Monographs, vol. 49, American Mathematical Society, Rhode Island, 1997. MR 98c:47076. Zbl 870.35004.

Luisa Arlotti: Dipartimento di Ingegneria Civile, via delle Scienze, 20833100 Udine, ITALY

E-mail address: Arlotti@dic.uniud.it 


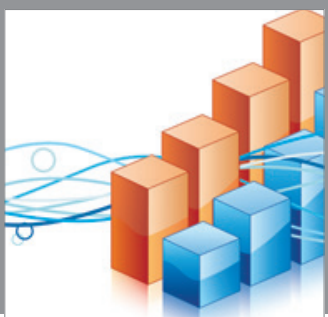

Advances in

Operations Research

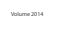

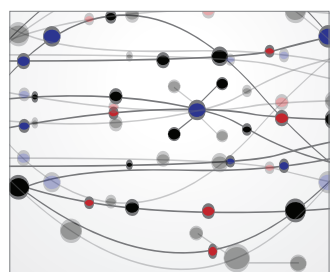

\section{The Scientific} World Journal
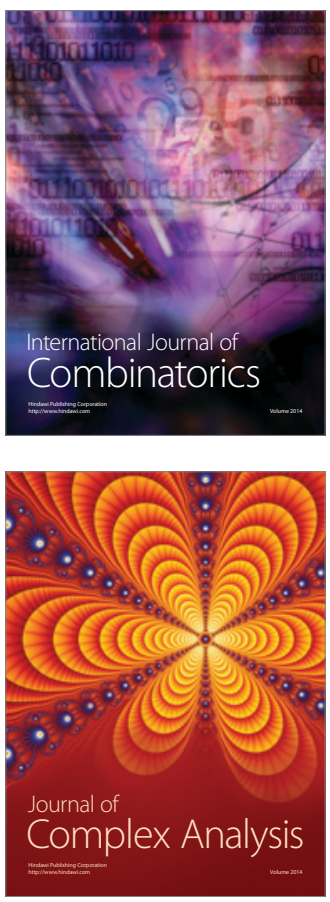

International Journal of

Mathematics and

Mathematical

Sciences
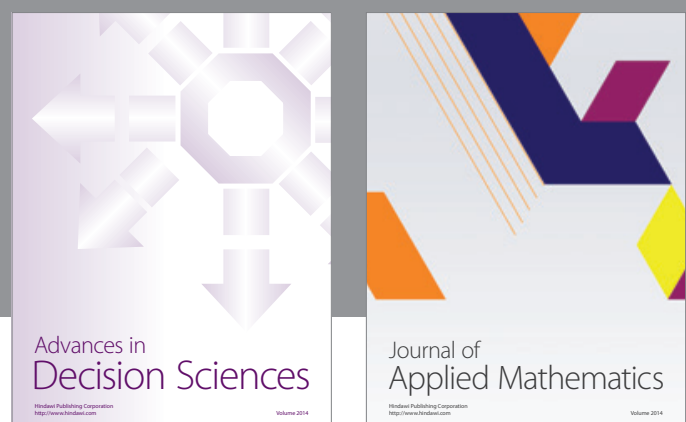

Journal of

Applied Mathematics
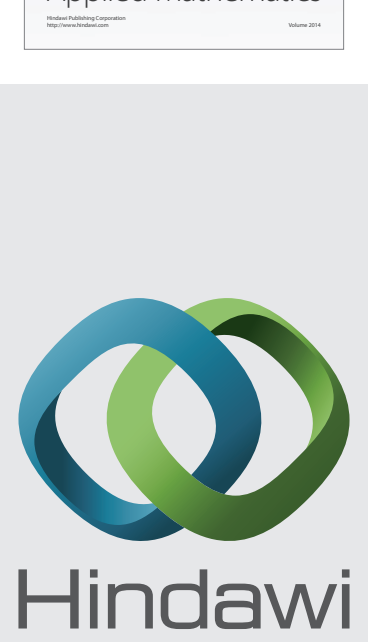

Submit your manuscripts at http://www.hindawi.com
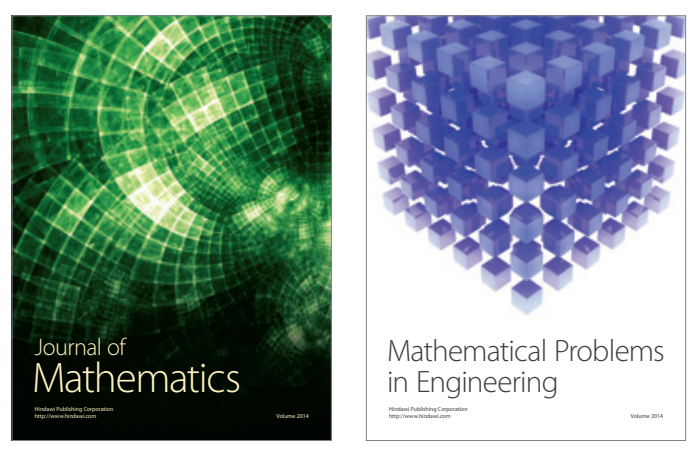

Mathematical Problems in Engineering
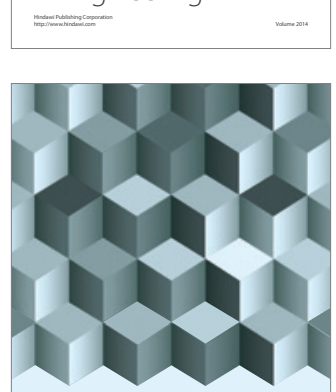

Journal of

Function Spaces
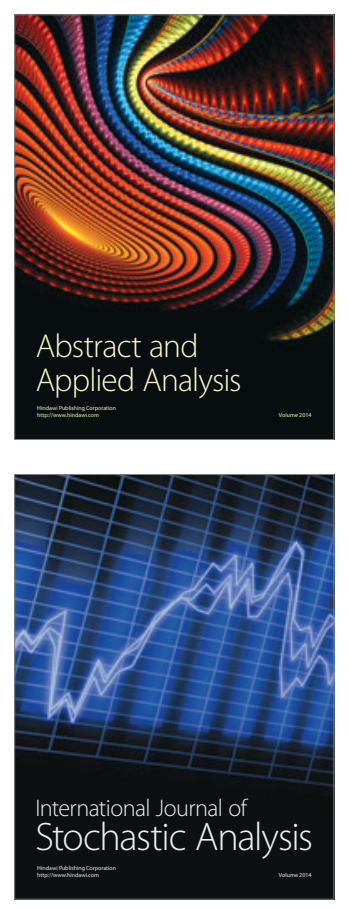

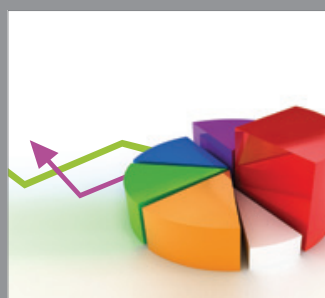

ournal of

Probability and Statistics

Promensencen
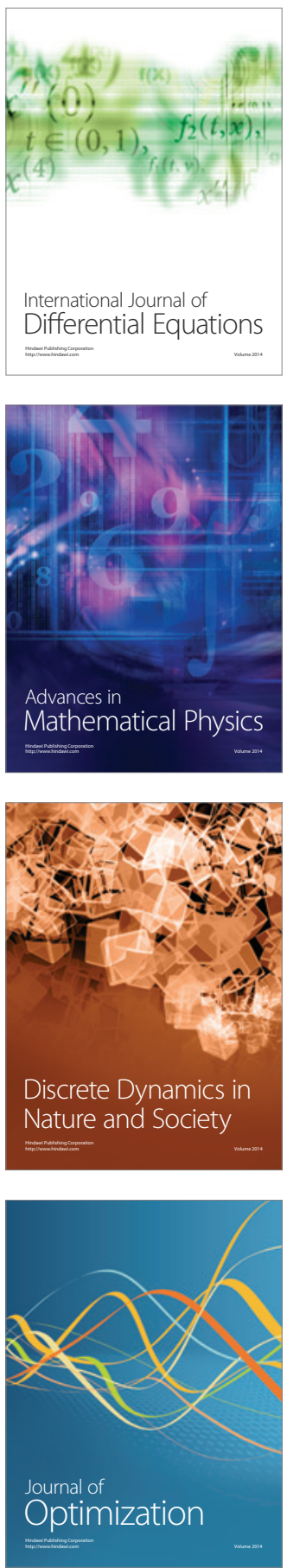